\title{
BMJ Open Efficacy of coupled plasma filtration adsorption (CPFA) in patients with septic shock: A multicenter randomised controlled clinical trial
}

\author{
Sergio Livigni, ${ }^{1}$ Guido Bertolini, ${ }^{2}$ Carlotta Rossi, ${ }^{2}$ Fiorenza Ferrari, ${ }^{1}$ \\ Michele Giardino, ${ }^{2}$ Marco Pozzato, ${ }^{3}$ Giuseppe Remuzzi, ${ }^{2}$ GiViTI: Gruppo Italiano \\ per la Valutazione degli Interventi in Terapia Intensiva (Italian Group for the \\ Evaluation of Interventions in Intensive Care Medicine) is an independent \\ collaboration network of Italian Intensive Care units
}

To cite: Livigni S, Bertolini G, Rossi C, et al. Efficacy of coupled plasma filtration adsorption (CPFA) in patients with septic shock: A multicenter randomised controlled clinical trial. BMJ Open 2014;4:e003536. doi:10.1136/bmjopen-2013003536

- Prepublication history and additional material for this paper is available online. To view these files please visit the journal online (http://dx.doi.org/10.1136/ bmjopen-2013-003536).

Received 17 July 2013 Revised 9 December 2013 Accepted 11 December 2013

CrossMark

For numbered affiliations see end of article.

Correspondence to Dr Guido Bertolini; guido.bertolini@marionegri.it

\section{ABSTRACT}

Objectives: Coupled plasma filtration adsorption (CPFA, Bellco, Italy), to remove inflammatory mediators from blood, has been proposed as a novel treatment for septic shock. This multicenter, randomised, nonblinded trial compared CPFA with standard care in the treatment of critically ill patients with septic shock.

Design: Prospective, multicenter, randomised, openlabel, two parallel group and superiority clinical trial.

Setting: 18 Italian adult, general, intensive care units (ICUs).

Participants: Of the planned 330 adult patients with septic shock, 192 were randomised to either have CPFA added to the standard care, or not. The external monitoring committee excluded eight ineligible patients who were erroneously included.

Interventions: CPFA was to be performed daily for 5 days, lasting at least $10 \mathrm{~h} /$ day.

Primary and secondary outcome measures: The primary endpoint was mortality at discharge from the hospital at which the patient last stayed. Secondary endpoints were: 90-day mortality, new organ failures and ICU-free days within 30 days.

Results: There was no statistical difference in hospital mortality ( $47.3 \%$ controls, $45.1 \%$ CPFA; $p=0.76)$, nor in secondary endpoints, namely the occurrence of new organ failures $(55.9 \%$ vs $56.0 \% ; p=0.99)$ or free-ICU days during the first 30 days $(6.8$ vs $7.5 ; p=0.35)$. The study was terminated on the grounds of futility. Several patients randomised to CPFA were subsequently found to be undertreated. An a priori planned subgroup analysis showed those receiving a CPFA dose $>0.18 \mathrm{~L} / \mathrm{kg} /$ day had a lower mortality compared with controls ( $O R$ 0.36, $95 \% \mathrm{Cl} 0.13$ to 0.99 ).

Conclusions: CPFA did not reduce mortality in patients with septic shock, nor did it positively affect other important clinical outcomes. A subgroup analysis suggested that CPFA could reduce mortality, when a high volume of plasma is treated. Owing to the inherent potential biases of such a subgroup analysis, this result can only be viewed as a hypothesis generator and should be confirmed in future studies.
ClinicalTrials.gov: NCT00332371; ISRCTN24534559.

\section{INTRODUCTION}

The host response against pathogens is a complex one. It is modulated through the production of numerous mediators that, among other mechanisms, promote proinflammatory and anti-inflammatory responses. ${ }^{1-4}$ The balance between these two pathways heavily influences the outcome. ${ }^{4-9}$ The amount and timing of release of different mediators, their relatively short half-lives, their limited range of action, their considerable redundancy and pleiomorphisms and the underexpression or overexpression of their receptors ${ }^{10-12}$ have negatively affected the numerous therapeutic attempts to neutralise specific molecules. ${ }^{12}$ The repeated failure of this strategy suggested a potentially greater utility may be achieved through simultaneous removal of several mediators to rebalance the immune response. This can be accomplished by various blood purification techniques, of which coupled plasma filtration adsorption (CPFA) can nonselectively remove the majority of soluble inflammatory mediators. ${ }^{13}$

Early experience with CPFA showed an increased survival in a rabbit model of endotoxin-induced septic shock. ${ }^{14}$ The first clinical study showed that a single treatment lasting $10 \mathrm{~h}$ significantly improved the haemodynamic status. ${ }^{15}$ These preliminary observations were confirmed in a study of 10 septic shock patients in whom norepinephrine requirements were progressively reduced and eventually discontinued after an average of 
five daily CPFA sessions, ${ }^{16}$ without adverse events. Subsequently, several Italian ICUs adopted CPFA in septic shock patients with promising results, and were willing to formally evaluate its efficacy. GiViTI, the Italian ICU network, thus launched a randomised multicentre clinical trial to assess the efficacy of CPFA in reducing mortality of critically ill patients with septic shock.

\section{METHODS}

Written consent was obtained from the patient when possible; otherwise physicians enroled patients according to the article 4.8.15 of the Guidelines for Good Clinical Practice. ${ }^{17}$.

\section{Setting and participants}

The study was performed in 18 adult ICUs who regularly used CPFA in the treatment of septic shock. Patients $>18$ years of age with septic shock either at or during their admission to the ICU were eligible for study entry, provided that CPFA could be started within $6 \mathrm{~h}$ from the occurrence of hypotension refractory to fluid resuscitation. This was made by the attending physician (present 24/7) using explicit criteria. ${ }^{18}$ Reasons for exclusion prior to randomisation were: pregnancy, cardiopulmonary resuscitation, coma $(\mathrm{GCS} \leq 8)$ due to an organic cerebral disease, metastatic cancer, contraindication to a haemopurification technique, an estimated life expectancy less than 2 weeks, prior inclusion in the study, admission from another ICU where the patient remained for $>24 \mathrm{~h}$ and lack of informed consent.

The Project Margherita electronic case report form (eCRF) was used for this study. ${ }^{19}{ }^{20}$ The core data included demographics, admission diagnoses, severity of infection on admission, comorbidities, location of the patient prior to ICU admission, surgical status, reasons for ICU admission, Simplified Acute Physiology Score II (SAPS II) variables ${ }^{21}$ on admission, organ failures and diseases occurring during their ICU stay, the severity of infection reached, major procedures and interventions and ICU and hospital outcomes. For enroled patients, their clinical conditions, including the sequential organ failure assessment (SOFA) score, ${ }^{22}$ the Risk of renal dysfunction, Injury to the kidney, Failure of kidney function, Loss of kidney function and End-stage kidney disease (RIFLE) criteria $^{22 a}$ for acute renal dysfunction and CPFA parameters were collected at the time of randomisation and then daily until ICU discharge or for a maximum of 21 days. Interventions to assure study homogeneity and quality are described in the online supplementary material.

\section{Randomisation and interventions}

Eligibility criteria were flagged up in real time by the eCRF, which prompted the clinician to enrol the patient or to register reasons for not doing so. Once enroled, the patients were randomly allocated by the eCRF on a $1: 1$ basis to either have CPFA added to the standard care, or not. A blocked randomisation schedule (randomly permuting blocks of four and six) ${ }^{23}$ was implemented in the eCRF, with stratification according to the centre and the presence of septic shock on admission. The allocation was securely saved in the database and revealed only once baseline additional data collection were completed. All these procedures were implemented to guarantee allocation concealment. ${ }^{24}$

\section{Coupled plasma filtration adsorption}

CPFA was developed to non-specifically remove larger mediators during systemic inflammation with an extracorporeal circuit consisting of a plasma filter, a resin cartridge and a high-flux dialyser. ${ }^{25}$

CPFA was performed with the use of a four-pump modular treatment (Lynda, Bellco, Mirandola, Italy) consisting of a plasma filter $\left(0.45 \mathrm{~m}^{2}\right.$ polyethersulfone $)$ and a following absorption on an unselective hydrophobic resin cartridge $(140 \mathrm{~mL}$ for $70 \mathrm{~g}$, with a surface of about $700 \mathrm{~m}^{2} / \mathrm{g}$ ) and a final passage of the reconstituted blood through a high-permeability $1.4 \mathrm{~m}^{2}$ polyethersulfone haemofilter, in which convective exchanges may be applied in a postdilution mode (figure 1 ). ${ }^{26}$

The postdilution reinfusion rate could be set up to $4 \mathrm{~L} / \mathrm{h}$. The blood flow was maintained between 150 and $200 \mathrm{~mL}$ min, while the plasma flow was controlled by a filtration fraction ranging from $10 \%$ to $18 \%$ of blood flow. ${ }^{27}$ More specifically, the filtration fraction should be set to $10 \%$ in the first hour and then it should be gradually increased to the target value of $18 \%$. The minimum volume of plasma treated per day should be $10 \mathrm{~L}$, corresponding to a blood flow of $150 \mathrm{~mL}$ min and a filtration fraction of $12 \%$.

The reinfusion solution, sterile and pyrogen-free, with bicarbonate buffer, contained the following composition (mmol L): Na 140, K 1.5, Ca 2, Mg 0.75, Cl 108, bicarbonate 35 , acetate 4 and glucose 5.55 .

All fluids were administered at room temperature. During treatment, the patient's temperature was to be maintained possibly within physiological limits, and anyway higher than $35^{\circ} \mathrm{C}$. The anticoagulation protocol is described in the online supplementary material.

According to the available clinical evidence, CPFA was to be repeated daily for the first 5 days, lasting at least $10 \mathrm{~h}$ each time, so that an average of $0.15 \mathrm{~L} / \mathrm{kg} /$ day of plasma should have been treated per day.

\section{Outcomes, follow-up and plan of analysis}

The primary endpoint was mortality at discharge from the hospital in which the patients were last treated. Thus, for patients transferred to another hospital, mortality was assessed at the discharge from the hospital in which the patients last stayed. To minimise the bias due to the decision to have the relative dying at home, patients discharged in a terminal condition (life expectancy $<2$ weeks as estimated by the attending physician) were considered to have died at the time of hospital discharge. The primary analysis was by intention-to-treat; 


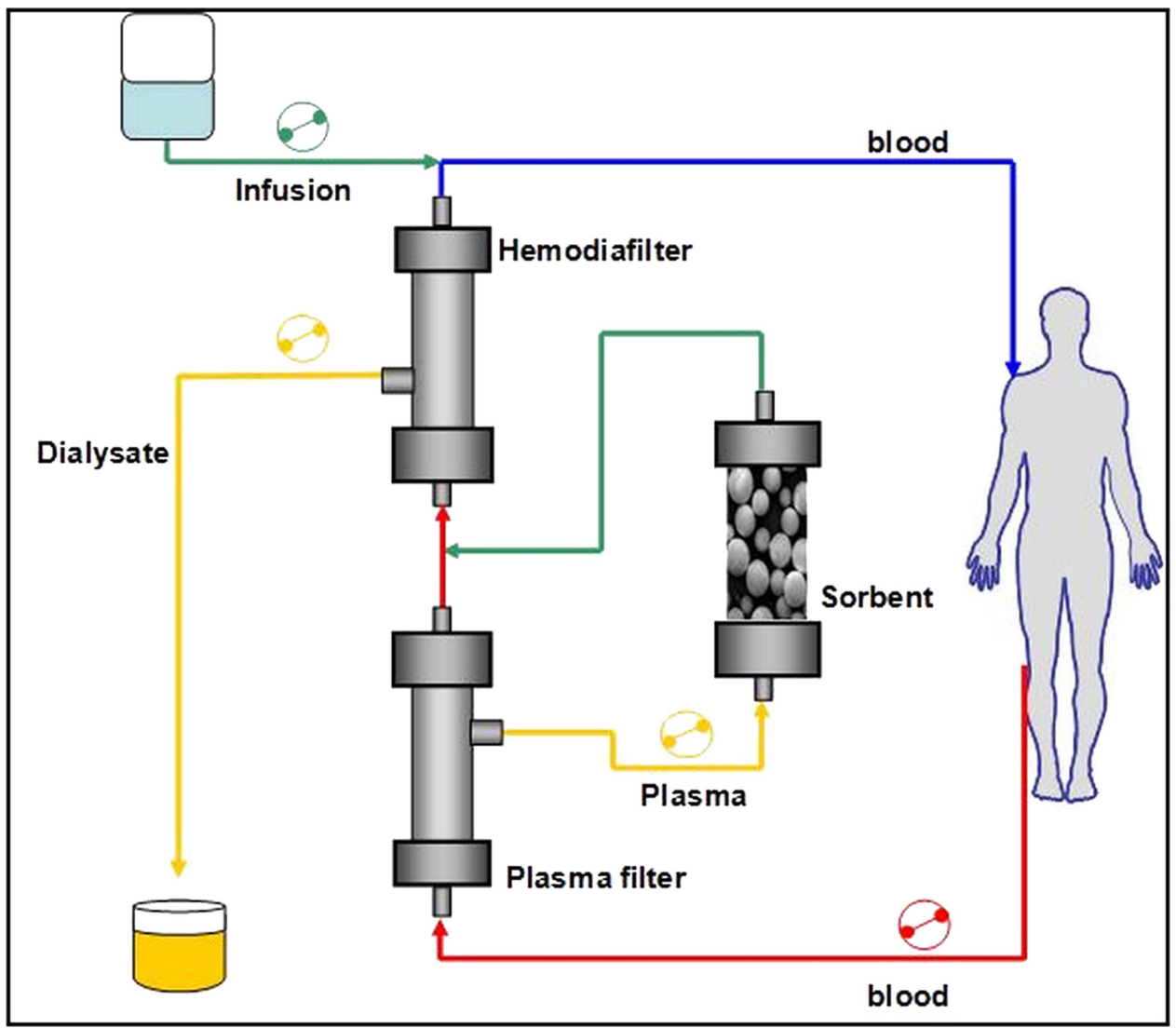

Figure 1 Coupled plasma filtration adsorption schema.

however, a per-protocol analysis was also planned to assess the impact of protocol violations, if any, on the primary endpoint. Secondary endpoints were: mortality within 90 days of randomisation; the proportion of patients who developed $\geq 1$ new organ failures during their ICU stay (defined by an organ SOFA score of 3 or $4^{22}$ ) and ICU-free days during the first 30 days from randomisation.

Timing of intervention is considered extremely important in septic shock. Thus, two subgroup analyses of the primary endpoint were pre-planned, namely the assessment of outcomes in patients with septic shock on ICU admission or who developed it during their ICU stay and patients starting CPFA within or later than $4 \mathrm{~h}$ of randomisation.

The study was sized to have $80 \%$ power to detect an improvement in hospital mortality from an expected $63 \%$ in the controls to $47 \%$ with CPFA (25\% relative improvement), with a two-tailed 5\% type I error. A total of 330 patients were required. A Bayesian approach (see online supplementary material) was adopted for interim analyses. ${ }^{23}$

\section{Premature termination of the trial}

In November 2010, the External Data and Safety Monitoring Committee (EDSMC) prompted an early termination of the study on the grounds of futility. To reach the a priori determined goal of a $25 \%$ reduction in mortality, in the second part of the study, a $23 \%$ hospital mortality in the CPFA group would have been required, which was considered implausible. Further concerns were the low recruitment rate and the high number of protocol violations in the CPFA arm in terms of low volume of plasma treated per day.

\section{Statistical analyses}

Hospital mortality was analysed using the $\chi^{2}$ test. The effect size was expressed in terms of absolute risk difference with its $95 \% \mathrm{CI}^{28}$ With regard to secondary endpoints and subgroup analyses, categorical variables were compared with $\chi^{2}$ or Fisher's exact tests, while a Student $\mathrm{t}$ test was used for continuous variables, after having assessed normality through the Kolmogorov-Smirnov, the Shapiro-Wilks tests and the normal probability plot, and homoscedasticity through the Levene's test. Mortality within 90 days of randomisation was assessed using Kaplan-Meier curves with any differences investigated through logrank testing.

As a number of protocol violations in the CPFA arm were registered due to a lower than planned volume of plasma treated, we also performed a per-protocol analysis of the primary endpoint, as determined a priori. The analysis by the 'adhesion to the protocol' was indeed planned to involve patients who did not have relevant protocol violations, to assess the possible influence of such violations on the outcome.

Hospital mortality was evaluated according to tertiles of the mean volume of plasma treated per $\mathrm{kg}$ per day. 
Any association between tertiles and hospital mortality was tested with the $\chi^{2}$ test and the Cochran-Armitage test for trend. As any benefit of randomisation was lost, comparison with the control group was performed through a logistic regression model that allowed to adjust for possible confounders (see online supplementary material).

\section{RESULTS}

Between January 2007 and November 2010, a total of 192 patients had been randomised. Recruitment in each ICU lasted a median of 22 months (IQR 13-26). During this period, 386 patients with septic shock were excluded as being non-eligible (see online supplementary material). Central monitoring subsequently identified 14 enroled patients whose eligibility criteria were doubtful. Further clinical information was retrieved and provided to the EDSMC who determined that eight of these patients (5 CPFA, 3 control) were erroneously enroled (see online supplementary material). Analysis was performed by intention-to-treat on the 184 remaining patients. ${ }^{29}$ Figure 2 denotes the flow of participants.
Table 1 shows the patients' characteristics; further details are provided in the online supplementary material. One episode of surgical wound bleeding was registered as possibly related to CPFA in a patient receiving drotrecogin alfa (activated).

Overall, 44 patients (48.4\%) had less than the minimum amount, as recommended by the protocol, of plasma treated over the first 5 days. They were evenly distributed across centres. To better express and investigate the phenomenon of undertreatment, and following the emerging concept of dose of renal replacement therapy, ${ }^{30}$ we computed the volume of plasma treated in $\mathrm{L} / \mathrm{kg} /$ day. In the 91 patients randomised in the CPFA arm, a mean of $0.15 \mathrm{~L} / \mathrm{kg} /$ day were treated for the first 5 days (tertiles $0.12-0.18$ ), and 0.18 for the first 3 days. Table 2 lists the reasons for undertreatment. Four patients died during CPFA, one before initiating the treatment, two at the very first moment and one after the first $0.09 \mathrm{~L} \mathrm{~kg}$ of plasma treated. The mean time to start CPFA after septic shock identification was $5.7 \mathrm{~h}$ (SD 3.8); 38 patients started within $4 \mathrm{~h}$. In the control group, in violation of the protocol, two patients were treated with CPFA; one died at 7 days postrandomisation, the other was discharged alive from the hospital 37 days after randomisation.

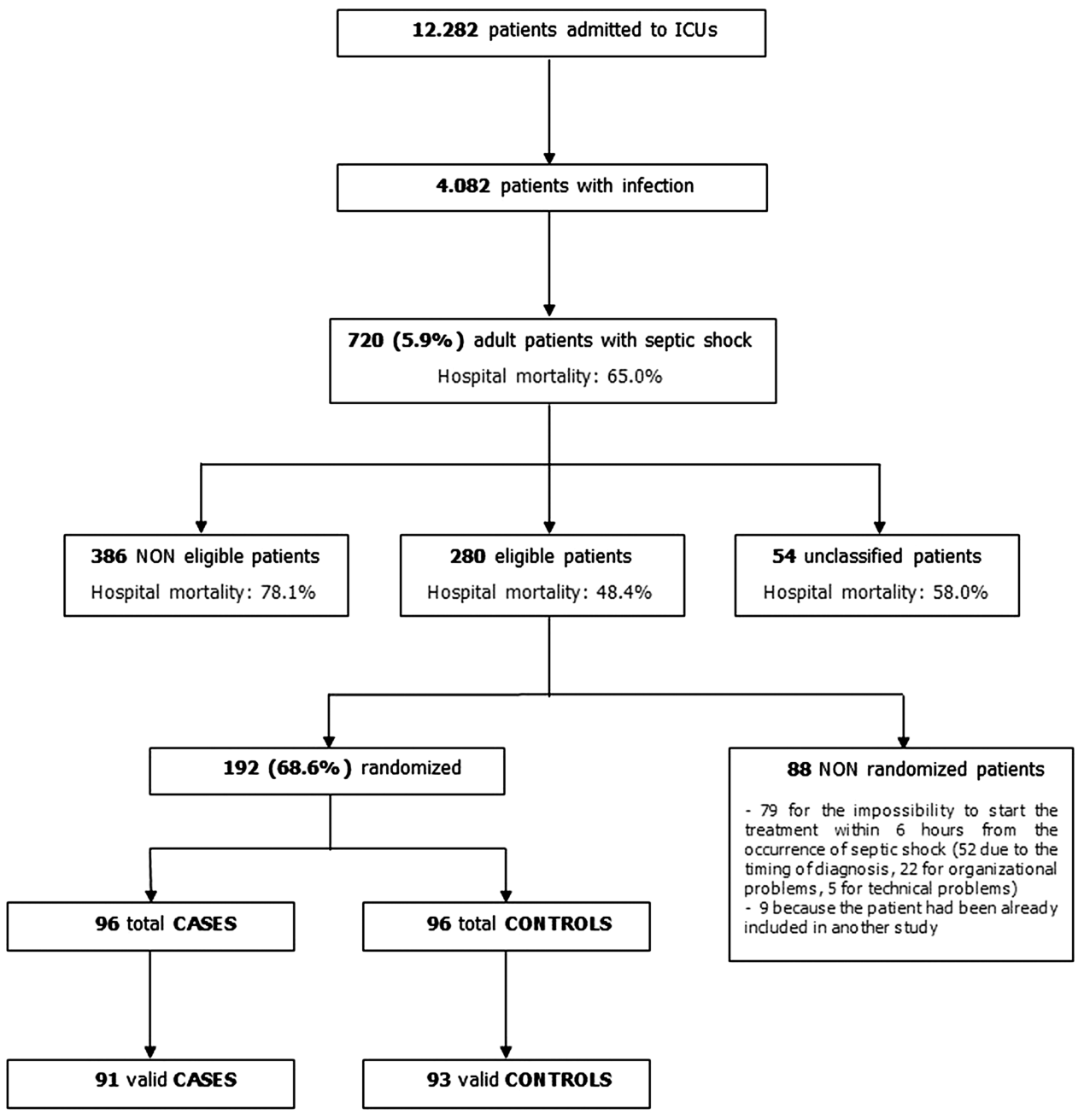

Figure 2 Flow chart of participants. 
Table 1 Characteristics of the patients before randomisation

\begin{tabular}{|c|c|c|}
\hline & Controls $(n=93)$ & CPFA ( $n=91)$ \\
\hline Sex (male), n (\%) & 65 (69.9) & $56(61.5)$ \\
\hline \multicolumn{3}{|l|}{ Age (years), n (\%) } \\
\hline Overall, mean [SD] & $64.9[13.3]$ & $63.6[14.4]$ \\
\hline $17-45$ & $10(10.8)$ & $9(9.9)$ \\
\hline $46-65$ & $34(36.6)$ & 35 (38.5) \\
\hline $66-75$ & $23(24.7)$ & 27 (29.7) \\
\hline$>75$ & $26(28.0)$ & $20(22.0)$ \\
\hline \multicolumn{3}{|l|}{ BMl (\%) } \\
\hline Underweight & $5(5.4)$ & $2(2.2)$ \\
\hline Normal weight & $34(36.6)$ & $27(29.7)$ \\
\hline Overweight & $24(25.8)$ & $31(34.1)$ \\
\hline Obese & 30 (32.3) & $31(34.1)$ \\
\hline Length of stay before ICU admission (days), mean [SD] & $6.5[13.8]$ & $6.2[11.8]$ \\
\hline \multicolumn{3}{|l|}{ Source of admission, $\mathrm{n}(\%)$} \\
\hline Emergency room & $16(17.2)$ & $31(34.1)$ \\
\hline Surgical ward & $43(46.2)$ & $31(34.1)$ \\
\hline Medical ward & $29(31.2)$ & $27(29.7)$ \\
\hline Other ICU & $5(5.4)$ & $2(2.2)$ \\
\hline \multicolumn{3}{|l|}{ Surgical status, $n(\%)$} \\
\hline Not surgical & $43(46.2)$ & 54 (59.3) \\
\hline Elective surgical & $8(8.6)$ & $6(6.6)$ \\
\hline Emergency surgical & $42(45.2)$ & $31(34.1)$ \\
\hline Trauma, $\mathrm{n}(\%)$ & $6(6.5)$ & $5(5.5)$ \\
\hline \multicolumn{3}{|l|}{ Comorbidities, n (\%) } \\
\hline None & $12(12.9)$ & $18(19.8)$ \\
\hline Mary Charlson Index, median [Q1-Q3] & $2[0-3]$ & $1[0-2]$ \\
\hline \multicolumn{3}{|l|}{ Reason for admission, $\mathrm{n}(\%)$} \\
\hline Monitoring/weaning & $7(7.5)$ & $7(7.7)$ \\
\hline Respiratory failures & $80(86.0)$ & $69(75.8)$ \\
\hline Cardiovascular failures & $50(53.8)$ & $58(63.7)$ \\
\hline Neurological failures & 12 (12.9) & 9 (9.9) \\
\hline Renal failure & $24(25.8)$ & $33(36.3)$ \\
\hline Multiple organ failures & 59 (63.4) & 65 (71.4) \\
\hline \multicolumn{3}{|l|}{ Top 3 non-infectious diseases on admission, $\mathrm{n}(\%)$} \\
\hline Metabolic disorder & $23(24.7)$ & $25(27.5)$ \\
\hline Gastrointestinal perforation & $16(17.2)$ & $15(16.5)$ \\
\hline ALI & $16(17.2)$ & $14(15.4)$ \\
\hline SAPS II on admission, median [Q1-Q3] & $53[43-67]$ & $51[42-65]$ \\
\hline SOFA at randomisation, median [Q1-Q3] & $9[8-11]$ & $9[8-11]$ \\
\hline \multicolumn{3}{|l|}{ RIFLE at randomisation, $\mathrm{n}(\%)$} \\
\hline No risk & $51(54.8)$ & 29 (31.9) \\
\hline Risk & $16(17.2)$ & $22(24.2)$ \\
\hline Injury & $10(10.8)$ & $21(23.1)$ \\
\hline Failure & $16(17.2)$ & $19(20.9)$ \\
\hline \multicolumn{3}{|l|}{ Septic shock on admission, $\mathrm{n}(\%)$} \\
\hline \multirow[t]{2}{*}{ Missing } & 39 (42.4) & $43(47.8)$ \\
\hline & 1 & 1 \\
\hline \multicolumn{3}{|l|}{ Site of infection, $n(\%)$} \\
\hline Pneumonia & $25(26.9)$ & $30(33.0)$ \\
\hline Peritonitis & $28(30.1)$ & $25(27.5)$ \\
\hline Primary bacteraemia & $1(1.1)$ & $8(8.8)$ \\
\hline Colecistitis/colangitis & $5(4.3)$ & $3(3.3)$ \\
\hline Urinary tract infection & $1(1.1)$ & $2(2.2)$ \\
\hline Other & $23(24.7)$ & $19(20.9)$ \\
\hline Multisite & $10(10.8)$ & $4(4.4)$ \\
\hline \multicolumn{3}{|l|}{ Top five microorganisms isolated, $n(\%)$} \\
\hline Non-ESBL producing Escherichia coli & $13(13.7)$ & $14(15.9)$ \\
\hline $\begin{array}{l}\text { Non-ESBL proaucing Escnericnia coll } \\
\text { Candida albicans }\end{array}$ & $4(4.2)$ & $6(6.8)$ \\
\hline
\end{tabular}


Table 1 Continued

\begin{tabular}{|c|c|c|}
\hline & Controls $(n=93)$ & CPFA $(n=91)$ \\
\hline Methicillin-resistant Staphylococcus aureus & $10(10.5)$ & $4(4.5)$ \\
\hline Penicillin sensitive Pneumococcus & $2(2.1)$ & $4(4.5)$ \\
\hline Ampicillin-resistant vancomycin-sensitive Enterococcus faecalis & $3(3.2)$ & $3(3.4)$ \\
\hline Gram-positive bacteria & $25(26.3)$ & $27(30.7)$ \\
\hline Gram-negative bacteria & $29(30.5)$ & $27(30.7)$ \\
\hline \multicolumn{3}{|c|}{$\begin{array}{l}\text { Q1-Q3=first and third quartiles; underweight=for male, BMI<20, for woman, BMl<19; normal weight=for man, BMI 20-25, for woman, BMI } \\
\text { 19-24; overweight=for male, BMI 25-30, for female, BMI 24-29; obese=for male, BMl }>30 \text {, for female, BMl>29; respiratory failure=need of } \\
\text { ventilatory support to maintain gas exchange; cardiovascular failure=need of vasoactive drugs to provide sufficient pump action; neurological } \\
\text { failures (GCS } \leq 8 \text { ); Renal failure=RIFLE score: injury or higher. } \\
\text { ALI, acute lung injury; BMI, body mass index; CPFA, coupled plasma filtration adsorption; ESBL, extended-spectrum } \beta \text {-lactamase; GCS, } \\
\text { Glasgow Coma Scale; ICU, intensive care unit; RIFLE, Risk of renal dysfunction, Injury to the kidney, Failure of kidney function, Loss of } \\
\text { kidney function and End-stage kidney disease; SAPS, simplified acute physiology score; SOFA, sequential organ failure assessment. }\end{array}$} \\
\hline
\end{tabular}

No statistical difference was found in hospital mortality with $47.3 \%$ dying in the control group (44/93) versus $45.1 \%$ dying in the CPFA group $(41 / 91, \mathrm{p}=0.76)$, with an absolute risk difference of $2.2 \%(95 \%$ CI $-12.2 \%$ to $16.6 \%$ ). The 90-day survival curves of the two groups substantially overlapped (logrank test, $\mathrm{p}=0.48$; figure 3 ). Secondary endpoints did not statistically differ: the occurrence of new organ failure was $55.9 \%$ in the control versus $56 \%$ for CPFA patients $(\mathrm{p}=0.99)$; the free ICU days during the first 30 days postrandomisation were 6.8 in the control group versus 7.5 in the CPFA group $(p=0.35)$. There were also no statistical differences in the a priori determined subgroups. Hospital mortality in patients with septic shock on ICU admission was comparable (16/39 (41\%) for control vs $19 / 43$ $(44.2 \%)$ for CPFA; $p=0.77)$. The same was observed for the subgroup of patients who developed septic shock during their ICU stay $(27 / 53(50.9 \%)$ control vs $21 / 47$ $(44.7 \%)$ CPFA; $p=0.53)$. Likewise, no statistical difference in mortality was observed between controls 44/93 $(47.3 \%)$, and patients starting CPFA within $4 \mathrm{~h}$ from randomisation $(17 / 38(44.7 \%) ; \mathrm{p}=0.88)$, nor in those who started CPFA after $4 \mathrm{~h}(20 / 46(43.5 \%) ; \mathrm{p}=0.76)$. In seven patients, the timing of CPFA initiation was missing. Eventually, no effect on the number of patients per ICU was observed.

The per-protocol analysis revealed a non-significant trend in hospital mortality according to the tertiles of volume of plasma treated per $\mathrm{kg}$ per day over the first 5 days (figure 4). The characteristics of the groups

Table 2 Reasons for undertreatment in the coupled plasma filtration adsorption arm $(n=44)$

\begin{tabular}{lrc}
\hline & n & Per cent \\
\hline Clotting of the circuit & 21 & 47.7 \\
Technical problems & 5 & 11.4 \\
Organisational problems & 4 & 9.1 \\
Patient's death & 4 & 9.1 \\
Lack of specialised personnel & 3 & 6.8 \\
Family request to stop CPFA & 1 & 2.3 \\
Other & 6 & 13.6 \\
\hline
\end{tabular}

defined by the tertiles are shown in the online supplementary material. The logistic regression model, aimed at adjusting for possible confounders, verified that hospital mortality in patients falling within the third tertile $(\geq 0.18 \mathrm{~L} / \mathrm{kg} /$ day of plasma treated over the first 5 days) was statistically lower than in the control group (OR 0.36 , 95\% CI 0.13 to 0.99 ; see table 3 ). We then performed two sensitivity analyses, namely limiting the evaluation of the volume of plasma treated to the first 3 days and excluding from the control and treated groups patients who died in the first $24 \mathrm{~h}$ postrandomisation. The first analysis was aimed at assessing whether any possible benefit of CPFA was obtained before 5 days; the second was intended to minimise any possible selection bias as patients who died early could not have entered the highest tertile of treated plasma due to insufficient time. Both sensitivity analyses (presented in the online supplementary material) confirmed the same estimates, even though statistical significance was lost for lack of power.

\section{DISCUSSION}

The prognosis of critically ill patients with septic shock remains poor, with mortality rates still around 50 $60 \%{ }^{20}{ }^{31}$ All attempts to find a 'magic bullet' to restore immune derangements during sepsis and improve the outcomes have failed, highlighting the complexity of the immune response, including a marked intrapatient variability in terms of magnitude of response, timing and trajectory and our continued lack of full understanding.

Rather than targeting a specific molecule, CPFA offered a more general means of reducing the circulating inflammatory mediator load. Following promising results in early phase studies, ${ }^{15} 16{ }^{25}$ GiViTI performed this randomised clinical trial to assess the efficacy of CPFA in reducing hospital mortality of patients affected by septic shock.

\section{The main findings}

After randomising more than half the planned number of patients, we found no statistical difference with the use of CPFA in hospital mortality, the occurrence of new organ failures or the overall clinical evolution. To 
Figure 3 Survival curves.

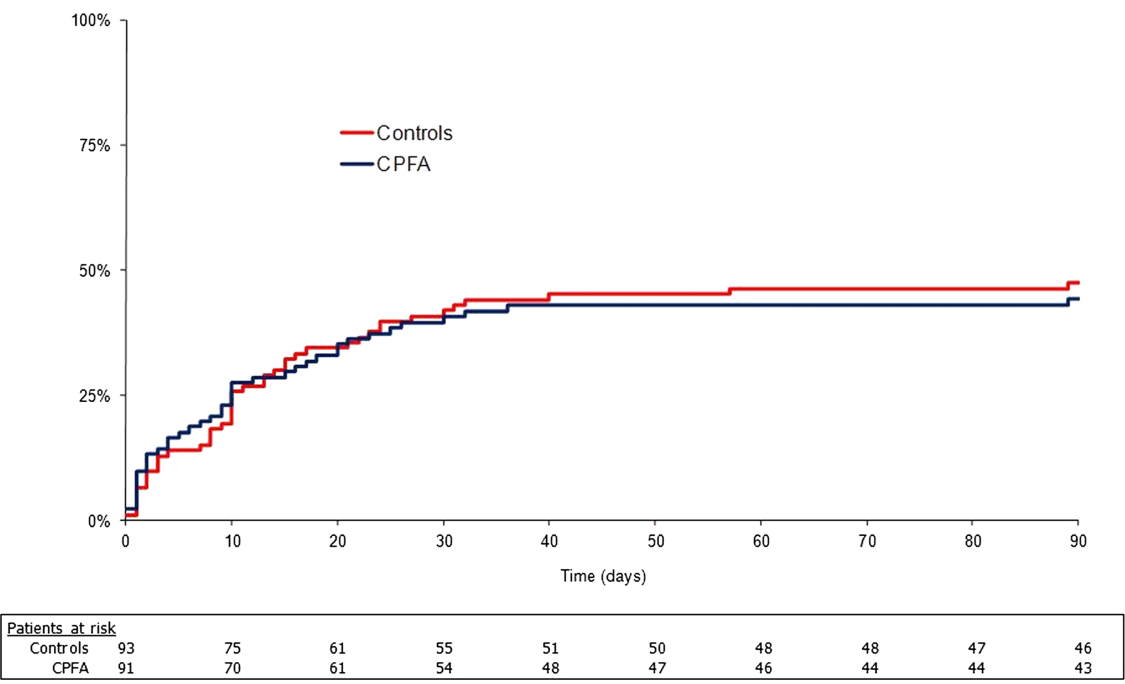

reverse these results, with the sample still to be randomised, implausible data should have been observed from then on. Furthermore, this study was powered from an anticipated $63 \%$ hospital mortality in the control group. Although such an estimation, coming from previous GiViTI data, was confirmed in the whole sample (figure 2), the eligibility criteria selected a subgroup where mortality was sensibly lower (47.3\%), thereby reducing the power of the study. Thus, the EDSMC considered that continuing to spend money in a clinical trial that had a little chance of demonstrating efficacy was undesirable and asked for a premature termination on the grounds of futility, although the anticipated, non-binding Bayesian futility criteria for stopping the trial were not fulfilled.

\section{The dilemma of primary endpoint}

The correct primary endpoint of clinical trials in septic shock is still debated. ${ }^{32}$ Most of the studies have adopted 28-day mortality due to Food and Drug Administration stipulations. However, the mortality rate attributable to sepsis continues long after the initiation of the acute event $^{33}$; indeed, $16.8 \%$ of our study patients were still in the ICU beyond 28 days after randomisation. On the other hand, overextending the follow-up period has the disadvantage of diluting the phenomenon, with the inclusion of competing causes of death. We thus considered mortality at the time of discharge from the hospital into which they were last admitted following their septic shock episode. At that point, the patient no longer requires aggressive, specialised, interdisciplinary care, which means he or she had survived the septic shock episode. A 90-day mortality was anyway recorded and considered as secondary endpoint.

\section{The problem of undertreatment}

Nearly half of the patients randomised to CPFA were undertreated as per protocol stipulation. This poses two
Figure 4 Hospital mortality according to the quantity of volume of plasma treated (whiskers represent 95\% Cl).

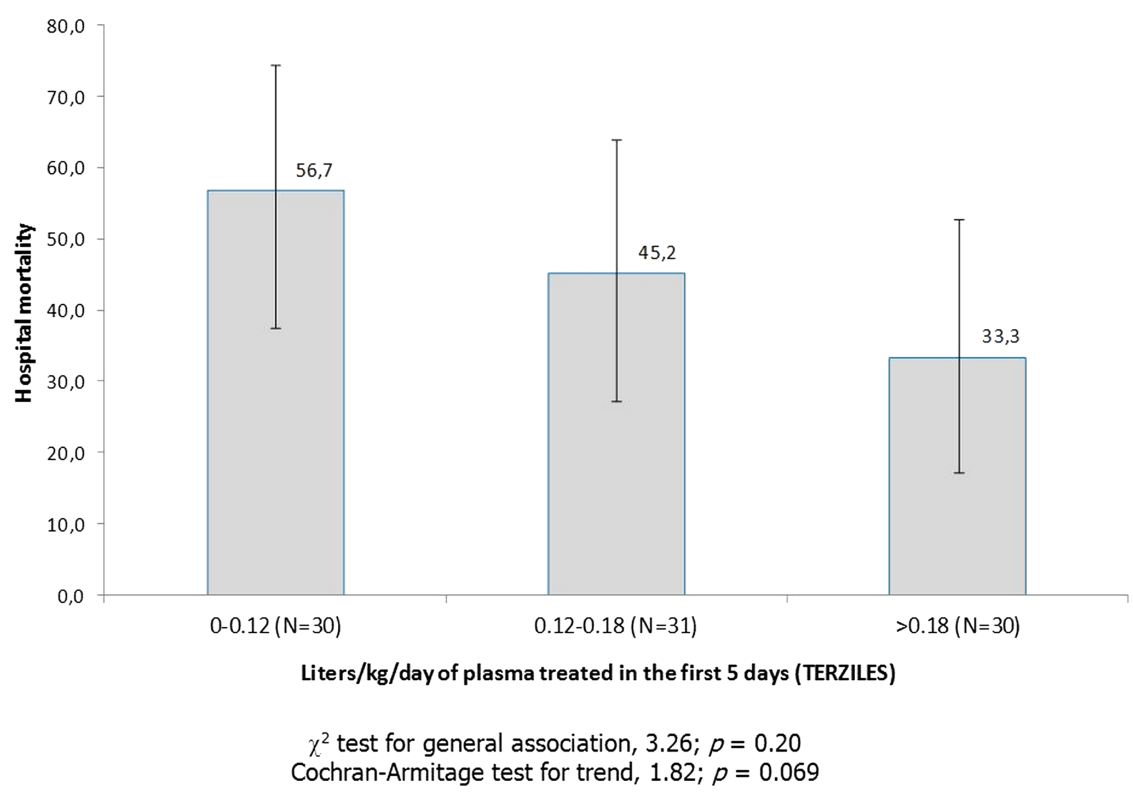


Table 3 Results of the logistic regression model on hospital mortality

\begin{tabular}{|c|c|c|c|}
\hline Variable & OR & $95 \% \mathrm{Cl}$ & p Value \\
\hline \multicolumn{4}{|l|}{ Volume of plasma treated (L/kg/day) } \\
\hline CPFA, $\leq 0.18\left(1^{\circ}\right.$ and $2^{\circ}$ tertiles $)$ vs controls & 1.52 & 0.73 to 3.17 & 0.033 \\
\hline CPFA, $>0.18\left(3^{\circ}\right.$ tertile $)$ vs controls & 0.36 & 0.13 to 0.99 & \\
\hline Age (decades) & 1.57 & 1.19 to 2.07 & 0.001 \\
\hline \multicolumn{4}{|l|}{ Source of admission } \\
\hline Other ICU vs medical ward & 0.28 & 0.04 to 1.89 & \\
\hline Emergency room vs medical ward & 0.27 & 0.11 to 0.67 & 0.021 \\
\hline Surgical ward vs medical ward & 0.34 & 0.15 to 0.77 & \\
\hline Renal failure at admission & 4.08 & 1.47 to 11.32 & 0.007 \\
\hline Cholecystitis or cholangitis on admission & 0.18 & 0.04 to 0.75 & 0.018 \\
\hline
\end{tabular}

crucial questions: the true feasibility of the technique in the ICU and the possible relationship between the overall negative result and such undertreatment. The main reason for not reaching the prescribed volume of plasma treated was clotting of the circuit (48\%). This problem was encountered by all centres.

Why did the training of the centres not have an effect? Many factors could have contributed. First, CPFA involves a complex circuit that includes a haemofilter, a plasma filter and an adsorbing cartridge, and requires an adequate balance of flows, dilutions and anticoagulation. We used heparin for anticoagulation (see online supplementary material), the most frequently used drug in this regard, because the machine used in the study did not support regional anticoagulation with citrate. Nevertheless, heparin is difficult to manage, particularly in the critically ill. Many centres may have been too conservative either with the heparin dosage and/or the blood flow rate through the circuit, or there may be an insufficient antithrombin substrate for the heparin to be effective. ${ }^{34}$ Second, because of the high cost of the procedure (about $€ 1.200$ per treatment), in most of the cases, the physicians did not start a new course of CPFA on the same day, in case of clotting of the circuit. Third, the training may have been (partly) ineffective. On the one hand, it only reached a few people per ICU. It was often difficult to involve the nephrologists, who, in many centres, are in charge of the procedure. On the other hand, despite excellent feedbacks from the participants, we cannot a posteriori exclude it was qualitatively suboptimal.

At any rate, the feasibility problems we have encountered in the present clinical trial suggest that the procedure, as implemented in this study, is not practicable in everyday clinical practice. Interestingly, regional anticoagulation with citrate represents a valid alternative to heparin as its anticoagulatory effect is limited to the extracorporeal circuit, without any systemic effect, and can be safely applied in the ICU. ${ }^{35} 36$ In a feasibility study carried out in 13 patients at high risk of bleeding, citrate regional anticoagulation was associated with a significantly lower number of clotted CPFA cartridges than with the heparin. ${ }^{37}$ The newer generation CPFA machine is able to apply citrate regional anticoagulation, and initial experiences in patients with septic shock demonstrate that a much higher volume of plasma can be safely treated. ${ }^{38}$ Should these preliminary results be confirmed, the question whether the reason of our negative result was a problem of feasibility or efficacy would become essential, to avoid the risk of dismissing a potentially effective treatment for such a high mortality condition as septic shock.

\section{The per-protocol analysis and its limits}

Of note, patients who had a larger volume of plasma treated seemed to have reduced hospital mortality. This cannot be taken as conclusive evidence of the efficacy of CPFA. Even though the per-protocol analysis was planned a priori with the expected direction of the effect being stated in advance, and a dose-response relationship was found, a number of potential problems threaten the validity of this result. First, a subgroup definition for the per-protocol analysis (ie, tertiles of plasma treated) was based on characteristics measured after randomisation. Under such circumstances, the allocation to a subgroup may have been influenced by the intervention in relation to the severity of the patient, causing an important bias. This would be the case, for example, if the probability of circuit clotting was higher in the more severely ill patients. Actually, the characteristics of the three subgroups were somewhat unbalanced (see online supplementary material). We adjusted for possible confounders in the multivariate model to minimise this risk, but we were limited to prognostic factors collected in the database. Particularly, we have no data on the immunoinflammatory status of the patients to account for. Second, the subgroup allocation may have been influenced by the outcome. For example, early deaths could have prevented the treatment of high volume of plasma. Even if we standardised the treated volume to the duration in hours of CPFA, since the treatment started with a low filtration fraction to be gradually 
increased to the target value (see online supplementary material), the first hours were characterised by a certain degree of undertreatment by design. In this case, an early death could have prevented the patient from being included in the third tertile, but not in the others, nor in the controls, spuriously influencing the result. We performed a sensitivity analysis by excluding early deaths from all groups, knowing that such an analysis could have greatly disadvantaged CPFA, if the lower number of early deaths were due to the efficacy of the technique. Interestingly, we verified that the strength of association was unchanged, albeit losing statistical significance for a lack of power, thereby excluding the presence of a differential outcome-related selection bias. Finally, the statistical significance of our results is quite thin; indeed, just one more death in the highest tertile subgroup would have rendered the difference in hospital mortality non-significant.

\section{Study limitations}

Almost $60 \%$ of patients with septic shock did not meet the inclusion criteria. The main reason was life expectancy less than 2 weeks. The mortality of these patients was in fact $98 \%$. Nonetheless, we cannot exclude that the higher severity could have brought about a potentially greater possibility of response to intervention, at least for some patients. Future studies should consider this aspect.

One-third of the eligible patients were not randomised due to the very narrow window $(6 \mathrm{~h})$ for the patient's recruitment and initiation of the treatment. This would have particularly hampered the generalisability of results had the findings been positive.

Finally, the study was terminated early for reasons of futility, after almost $60 \%$ of the originally planned patients had been recruited. This reduced the possibility of studying phenomena emerging from the analyses with a significant power, as in the case of the volume of plasma treated. In any event, any subgroup analysis, regardless of the involved sample size, could only have generated hypotheses. Our interpretation of the findings is in itself a hypothesis, which would have been only more robust with a larger sample.

\section{CONCLUSION}

CPFA was not able to reduce mortality in patients with septic shock. This result strongly discourages the use of CPFA in the everyday clinical practice, as it was implemented in this study. Unfortunately, we were not able to discern whether the culprit of such a negative result was the lack of effectiveness (mainly due to widespread feasibility problems) rather than the lack of true efficacy. The subgroup analysis was suggestive of efficacy, if a high volume of plasma was treated. Although we have taken counter measures to minimise potential biases, these cannot be completely excluded. Hence, this result can only be viewed as hypothesis generating. Given the new availability of citrate regional anticoagulation, we have designed a confirmatory, adaptive trial whose first step will be to prove this new technique easily allows high volume of plasma treated with CPFA.

\section{Author affiliations}

${ }^{1}$ Servizio Anestesia e Rianimazione B-DEA, Ospedale San Giovanni Bosco, Torino, Italy

${ }^{2}$ IRCCS-Istituto di Ricerche Farmacologiche "Mario Negri": Centro di Ricerche Cliniche per le Malattie Rare Aldo e Cele Daccò, Ranica, Bergamo, Italy

${ }^{3}$ Servizio di Nefrologia e Dialisi, Ospedale San Giovanni Bosco, Torino, Italy

Collaborators Armando Alborghetti (Ponte San Pietro-BG); Bruno Balicco (Zingonia-BG); Franco Bonello (Ivrea-TO); Francesco Casino (Matera-MT); Giacomo Castiglione (Catania-CT); Marco Cavana (Aosta-AO); Paolo Conti (Firenze-FI); Tiziana D’Amato (Imperia-IM); Carlo Donadio (Pisa-PI); Emilio Fabbri (Forlì-FC); Fiorenza Ferrari (Torino-TO); Bertilla Fiorese (Brescia-BS); Mario Gaggiotti (Brescia-BS); Marco Lorenz (Zingonia-BG); Mariella Maio (Torino-TO); Massimo Manes (Aosta-A0); Marco Manganaro (Alessandria-AL); Valerio Mangani (Firenze-FI); Antonio Mannarino (Firenze-FI); Gianmariano Marchesi (Bergamo-BG); Paolo Martinelli (Firenze-FI); Agnese Meterangelis (Ponte San Pietro-BG); Giulio Mingardi (Bergamo-BG); Giuseppe Nardi (Roma-RM); Antonella Peralta (Sanremo-IM); Marco Pozzato (Torino-TO); Marco Riggio (Lecco-LC); Francesco Massimo Romito (Matera-MT); Rosa Salcuni (Ivrea-T0); Silvano Scaioli (Forlì-FC); Silvia Scarrone (Alessandria-AL); Mario Tavola (Lecco-LC); Marina Terzitta (Forli-FC); Ernesto Turello (Alessandria-AL); Bruno Viaggi (Pisa-PI); Loretta Zambianchi (Forlì-FC).

Contributors All the authors substantially contributed to the conception and design interpretation of data, critically revising it and approved the final version of the manuscript, $\mathrm{GB}$ and $\mathrm{CR}$ were involved in the analysis of the data and $G B$ involved in the drafting the article.

Funding This work was supported by the Bellco SpA. The study was funded by GiViTI and through an unconditional research grant from Bellco (Mirandola, Italy), the CPFA patent holder, who had no role in the study. GiViTI-Istituto di Ricerche Farmacologiche Mario Negri has full ownership of the data and of the dissemination policy of the results. GiViTI is the recipient of unconditional grants from Brahms and Astellas, who also had no role in this study.

Competing interests None.

Ethics approval Ethics Committee of all centers.

Provenance and peer review Not commissioned; externally peer reviewed.

Data sharing statement The full protocol is accessible at: http://www.giviti. marionegri.it/COMPACT.asp Guido Bertolini and Carlotta Rossi had full access to all study data and take responsibility for its integrity and the accuracy of data analysis. Further analyses will be provided on request to the corresponding author. Raw data are available on justified request.

Open Access This is an Open Access article distributed in accordance with the Creative Commons Attribution Non Commercial (CC BY-NC 3.0) license, which permits others to distribute, remix, adapt, build upon this work noncommercially, and license their derivative works on different terms, provided the original work is properly cited and the use is non-commercial. See: http:// creativecommons.org/licenses/by-nc/3.0/

\section{REFERENCES}

1. Feezor RJ, Oberholzer C, Baker HV, et al. Molecular characterization of the acute inflammatory response to infections with gram-negative versus gram-positive bacteria. Infect Immun 2003;71:5803-13.

2. Bozza FA, Salluh JI, Japiassu AM, et al. Cytokine profiles as markers of disease severity in sepsis: a multiplex analysis. Crit Care 2007;11:R49.

3. Kellum JA, Kong L, Fink MP, et al. Understanding the inflammatory cytokine response in pneumonia and sepsis: results of the Genetic 
and Inflammatory Markers of Sepsis (GenIMS) Study. Arch Intern Med 2007;167:1655-63.

4. Angus DC, van der Poll T. Severe sepsis and septic shock. $N$ Engl $J$ Med 2013;369:840-51.

5. Bochud PY, Calandra T. Pathogenesis of sepsis: new concepts and implications for future treatment. BMJ 2003;326:262-6.

6. Weighardt $\mathrm{H}$, Heidecke $\mathrm{CD}$, Emmanuilidis $\mathrm{K}$, et al. Sepsis after major visceral surgery is associated with sustained and interferon-gamma-resistant defects of monocyte cytokine production. Surgery 2000;127:309-15.

7. Tamayo E, Fernandez A, Almansa R, et al. Pro- and anti-inflammatory responses are regulated simultaneously from the first moments of septic shock. Eur Cytokine Netw 2011;22:82-7.

8. Annane D, Bellissant E, Cavaillon JM. Septic shock. Lancet 2005;365:63-78.

9. Hotchkiss RS, Karl IE. The pathophysiology and treatment of sepsis. N Engl J Med 2003;348:138-50.

10. Cavaillon JM, Annane D. Compartmentalization of the inflammatory response in sepsis and SIRS. J Endotoxin Res 2006;12:151-70.

11. Wang ZM, Liu C, Dziarski R. Chemokines are the main proinflammatory mediators in human monocytes activated by Staphylococcus aureus, peptidoglycan, and endotoxin. $\mathrm{J} \mathrm{Biol} \mathrm{Chem}$ 2000;275:20260-7.

12. Munoz C, Misset B, Fitting C et al. Dissociation between plasma and monocyte-associated cytokines during sepsis. Eur J Immunol 1991;21:2177-84.

13. Tetta $\mathrm{C}$, Bellomo $\mathrm{R}$, Inguaggiato $\mathrm{P}$, et al. Endotoxin and cytokine removal in sepsis. Ther Apher 2002;6:109-15.

14. Tetta C, Gianotti L, Cavaillon JM, et al. Coupled plasma filtration-adsorption in a rabbit model of endotoxic shock. Crit Care Med 2000;28:1526-33

15. Ronco C, Brendolan A, Lonnemann G, et al. A pilot study of coupled plasma filtration with adsorption in septic shock* ${ }^{*}$ Crit Care Med 2002;30:1250-5.

16. Formica M, Olivieri C, Livigni S, et al. Hemodynamic response to coupled plasmafiltration-adsorption in human septic shock. Intensive Care Med 2003;29:703-8.

17. Human Medicines Evaluation Unit. Guidelines for good clinical practice. London: European Agency for the Evaluation of Medical Products, 1996:17-20.

18. Levy MM, Fink MP, Marshall JC, et al. 2001 SCCM/ESICM/ACCP/ ATS/SIS International Sepsis Definitions Conference. Intensive Care Med 2003;29:530-8.

19. Boffelli S, Rossi C, Anghileri A, et al. Continuous quality improvement in intensive care medicine. The GiViTI Margherita Project-Report 2005. Minerva Anestesiol 2006;72:419-32.

20. Malacarne $\mathrm{P}$, Langer M, Nascimben $\mathrm{E}$, et al. Building a continuous multicenter infection surveillance system in the intensive care unit: findings from the initial data set of 9,493 patients from 71 Italian intensive care units. Crit Care Med 2008;36:1105-13.

21. Le Gall JR, Lemeshow S, Saulnier F. A new Simplified Acute Physiology Score (SAPS II) based on a European/North American multicenter study. JAMA 1993;270:2957-63.
22. Vincent JL, de Mendonca A, Cantraine F, et al. Use of the SOFA score to assess the incidence of organ dysfunction/failure in intensive care units: results of a multicenter, prospective study. Working group on "sepsis-related problems" of the European Society of Intensive Care Medicine. Crit Care Med 1998;26:1793-800.

22a. Bellomo R, Ronco C, Kellum JA, et al. Acute renal failure definition, outcome measures, animal models, fluid therapy and information technology needs: the Second International Consensus Conference of the Acute Dialysis Quality Initiative (ADQI) Group. Crit Care 2004;8:R204-12.

23. Piantadosi S. Clinical trial. A methodological perspective. New York: Jonh Wiley \& Sons, 1997

24. Schulz KF, Grimes DA. Allocation concealment in randomised trials: defending against deciphering. Lancet 2002;359:614-18.

25. Page M, Rimmele T. [Coupled plasma filtration adsorption: rationale and perspectives in septic shock]. Can $J$ Anaesth 2008:55:847-52.

26. Ronco C, Brendolan A, d'Intini V, et al. Coupled plasma filtration adsorption: rationale, technical development and early clinical experience. Blood Purif 2003;21:409-16.

27. Formica $\mathrm{M}$, Inguaggiato $\mathrm{P}$, Bainotti $\mathrm{S}$, et al. Coupled plasma filtration adsorption. Contrib Nephrol 2007;156:405-10.

28. Kleinbaum D, Kupper L, Morgenstern H. Epidemiologic research New York, NY: Van Nostrand Reynhold, 1982

29. Fergusson D, Aaron SD, Guyatt G, et al. Post-randomisation exclusions: the intention to treat principle and excluding patients from analysis. BMJ 2002;325:652-4.

30. Prowle JR, Schneider A, Bellomo R. Clinical review: optimal dose of continuous renal replacement therapy in acute kidney injury. Crit Care 2011;15:207.

31. Annane D, Aegerter $\mathrm{P}$, Jars-Guincestre MC, et al. Current epidemiology of septic shock: the CUB-Rea Network. Am J Respir Crit Care Med 2003;168:165-2.

32. Cohen J, Guyatt G, Bernard GR, et al. New strategies for clinical trials in patients with sepsis and septic shock. Crit Care Med 2001;29:880-6.

33. Perl TM, Dvorak L, Hwang $T$, et al. Long-term survival and function after suspected gram-negative sepsis. JAMA 1995;274:338-45.

34. Singer M, McNally T, Screaton G, et al. Heparin clearance during continuous veno-venous haemofiltration. Intensive Care Med 1994;20:212-15.

35. Zhang Z, Hongying N. Efficacy and safety of regional citrate anticoagulation in critically ill patients undergoing continuous renal replacement therapy. Intensive Care Med 2012;38:20-8.

36. Mariano F, Bergamo D, Gangemi EN, et al. Citrate anticoagulation for continuous renal replacement therapy in critically III patients: success and limits. Int J Nephrol 2011;2011:748320. 1-5.

37. Mariano F, Tetta C, Stella M, et al. Regional citrate anticoagulation in critically ill patients treated with plasma filtration and adsorption. Blood Purif 2004;22:313-19.

38. Pozzato M, Ferrari F, Cecere P, et al. Safety and efficacy of citrate anticoagulation in spetic shock patients treated with couplet plasma filtration adsorbtion (CPFA). J Am Soc Nephrol 2011;22-Congress Proceeding:605A 